\title{
COMPARISON OF ARTIFICIAL HYDROPHILIC AND LIPOPHILIC MEMBRANES AND HUMAN SKIN TO EVALUATE NIACINAMIDE PENETRATION IN VITRO
}

\author{
ANNA NOWAK ${ }^{1}$, MARTIN K. CHURCH ${ }^{3}$, WIKTORIA DUCHNIK', \\ MONIKA RÓŻEWICKA-CZABAŃSKA², STANISŁAWA BIELECKA-GRZELA', \\ PIOTR PROWANS ${ }^{4}$, JAN PETRICZKO ${ }^{4}$, NORBERT CZAPLA ${ }^{4}$, \\ PIOTR BARGIEL ${ }^{4}$ and ADAM KLIMOWICZ ${ }^{1 *}$
}

'Department of Cosmetic and Pharmaceutical Chemistry, Pomeranian Medical University in Szczecin, PL-70111 Szczecin, Poland

${ }^{2}$ Clinic of Skin and Venereal Diseases, Pomeranian Medical University in Szczecin, PL-72010 Police, Poland ${ }^{3}$ Department of Dermatology, Charité - Universitätsmedizin Berlin, Chariteplatz 1, D-10117 Berlin, Germany

${ }^{4}$ Clinic of Plastic, Endocrine and General Surgery, Pomeranian Medical University in Szczecin, PL-72010 Police, Poland

\begin{abstract}
The evaluation of skin penetration of active substances from topical preparations is a pre-clinical requirement in the pharmaceutical industry. While in vitro preparations of human or animal skin are frequently used the development of artificial lipophilic and hydrophilic membranes with similar properties to human or animal skin needs to be developed. This preliminary study compared the in vitro permeation of niacinamide (vitamin B3), which is used as a model drug, across artificial hydrophilic and lipophilic membranes with in vitro penetration across human skin. The experiments were carried out in Franz diffusion cells. Artificial membranes were filter paper impregnated with either collodium (hydrophilic), stearic acid, cetyl alcohol or cetyl alcohol + cholesterol (lipophilic). Hydrogel and emulsion containing $0.5 \%$ of niacinamide were applied to membranes for up to 4 hours. Acidic and neutral (pH 5.4 and 7.4) acceptor fluid samples were taken after 0.5, 1, 2 and 4 hours. Niacinamide permeated hydrophilic better than lipophilic membranes. There were no significant differences between the cumulated amounts of niacinamide that penetrated artificial lipophilic membranes consisting of stearic acid, cetyl alcohol and cholesterol as compared with human skin in vitro. Also, hydrogel and emulsion gave similar results. In conclusion, these artificial membranes seem to be a promising alternative to human or animal skin.
\end{abstract}

Keywords: Niacinamide (Vitamin B3), penetration, Franz diffusion cell, artificial membrane, human skin

Abbreviations: human skin - HS, cellulose acetate - CA, collodium - Col, collodium/cetyl alcohol $\mathrm{Col} / \mathrm{CetAl}$, collodium/cetyl alcohol/cholesterol - Col/CetAl/Chol, collodium/stearic acid - Col/St acid, PBS phosphate buffered saline

Assessment of skin penetration of topically applied drugs is an important problem in the development of dermal creams and ointments. To evaluate this in humans in vivo, several models have been developed including suction blister fluid, tape-stripping and cutaneous microdialysis (1). However, such methods are somewhat invasive, so in vitro techniques based on the application of substances to human or animal skin have been developed. As human skin is not easily available, the use of artifi- cial membranes, both lipophilic and/or hydrophilic, seems to be a possible alternative.

The penetration of active substances through the skin is dependent on a number of factors including a structure, molecular size and molecular weight as well as on other physical and chemical properties, especially its water and lipid solubility. After topical application, the first important barrier encountered is the stratum corneum, which protects the skin against harmful external factors, decreasing the pen-

\footnotetext{
* Corresponding author: e-mail: adklim@pum.edu.pl
} 
etration efficiency and reducing the absorption of exogenous compounds put on skin $(2,3,4,5)$. The stratum corneum is built of 10-15 layers of dead, flattened corneocytes, which are subject to permanent exfoliation. The intercellular spaces are filled with lipids forming about $10 \%$ of the mass of the layer. They consist of ceramides, cholesterol, fatty acids, cholesterol esters and small amounts of phospholipids $(6,7)$.

In order to avoid the use of human or animal skin, a number of artificial membranes have been developed which imitate the stratum corneum. A further property of an artificial membrane is an adequate composition of lipid substances that resemble those naturally occurring in the skin. Up to now, the following membranes, among others, were used: PVPA (Phospholipid Vesicle-based Permeation Assay), the composition of which resembles stratum corneum $(8,9)$, PAMPA (Parallel Artificial Membrane Permeability Assay) membranes (9, 10, 11) and other lipophilic membranes containing cholesterol, stearic acid and silicone oil $(12,13,14)$. In addition, hydrophilic membranes that resemble the hydrophilic characters of the deeper part of skin composed of cellulose nitrate or acetate have been used $(14,15,16,17)$.

Vitamin $B_{3}$ is composed of nicotinic acid amide (niacinamide), nicotinic acid (niacin) and its esters (Fig. 1). Niacinamide is a water-soluble compound of molecular weight $122.1 \mathrm{Da}$ and melting point $130^{\circ} \mathrm{C}$, its $\mathrm{pK}_{\mathrm{a}}$ is 3.35 at $20^{\circ} \mathrm{C}$, partition coefficient octanol/water is -0.35 . This substance is widely applied in cosmetic preparation at a concentration of $2-5 \%$. Niacinamide is a well-tolerated and safe compound with a good bioavailability $(11,18,19)$.

It is characterized by anti-inflammatory properties; therefore, it can be applied, inter alia, in atopic dermatitis and acne rosacea. Moreover, it reduces sebum excretion $(20,21,22)$, protecting the skin against photoaging, so it may be a non-toxic alternative in melanoma chemoprevention in highrisk individuals $(19,23,24)$. Thus, it can be applied in pharmaceutical and cosmetic products.
A<smiles>O=C(O)c1cccnc1</smiles>

B<smiles>NC(=O)c1cccnc1</smiles>

Figure 1. Molecular structure of nicotinic acid (A) and nicotinamide (B).
The aim of the study was to evaluate the in vitro permeation across artificial hydrophilic and lipophilic membranes of niacinamide applied as hydrogel and emulsion, as compared with in vitro human skin penetration.

\section{EXPERIENTIAL}

\section{Materials}

Niacinamide (p.a.), cetyl alcohol (p.a.), polyoxyethylene sorbitan trioleate (Tween 85) were purchased from Sigma-Aldrich, USA, disodium phosphate (p.a.), potassium dihydrogen phosphate (p.a.) and n-octanol (p.a.) - from Merck (Germany); acetonitrile from J.T. Baker, (the Netherlands), cholesterol from Coel, Kraków (Poland), stearic acid (p.a.) and glycerol (p.a.) from Chempur (Poland); collodion from Caelo Caesar \& Lorenz GmbH (Germany); biobase (consisted of glyceryl stearate, cetearyl alcohol and sodium stearoyl lactylate), cocoa butter, grape seed oil, hydroxyethylcellulose from Mazidła (Poland), and cellulose acetate membranes from Sartorius Stedim Biotech GmbH (Germany).

\section{Human skin}

Human abdominal skin obtained after plastic surgery was used. The research was approved by the ethical committee of Pomeranian Medical University in Szczecin. Written informed consent to use their skin was obtained from all donors.

The skin of $0.5 \mathrm{~mm}$ in thickness was dermatomed. Afterward, the skin was divided into pieces of $2 \times 2 \mathrm{~cm}$ and washed in PBS (phosphate-buffered saline) ( $\mathrm{pH} \mathrm{7.4)}$ for $30 \mathrm{~min}$. The skin was then wrapped in aluminum foil and stored at $-20^{\circ} \mathrm{C}$ until use for a maximum of 6 months. Under these conditions, the skin is stable with regard to its barrier properties (24). Prior to the experiments, the skin samples were thawed at room temperature for about $30 \mathrm{~min}$.

\section{In vitro permeation}

All experiments were performed in Franz diffusion cells (SES Analyse Systeme GmbH, Germany) composed of a donor chamber of $2 \mathrm{~mL}$ capacity and an acceptor chamber of $8 \mathrm{~mL}$ capacity. The penetration surface was $1 \mathrm{~cm}^{2}$. As acceptor fluids, $0.1 \mathrm{M}$ phosphate buffers of two different $\mathrm{pH}$ values, 5.4 (similar to that of skin surface) and 7.4 (comparable to $\mathrm{pH}$ of deeper skin layers) were used. In the acceptor chamber, a constant temperature of $37^{\circ} \mathrm{C} \pm 1^{\circ} \mathrm{C}$ was maintained via a thermostat. The acceptor fluid was mixed with a magnetic stirrer.

The study was conducted for 4 hours. After $0.5,1,2$ and $4 \mathrm{~h}$ an aliquot of $0.3 \mathrm{~mL}$ of acceptor 
fluid was taken. Thereafter the same volume of fresh buffer of the same $\mathrm{pH}$ was added to the acceptor fluid chamber.

To evaluate niacinamide (NA) penetration, commercial cellulose acetate membranes (CA), pore size $0.2 \mu \mathrm{m}$, as well as artificial, both hydrophilic and lipophilic, membranes made in our laboratory were used. To obtain hydrophilic membranes, pieces of filter paper, $2.5 \mathrm{~cm}$ in diameter, were impregnated with a fixed volume of collodion, consisting mainly of cellulose nitrate in diethyl ether - ethanol solution (Col). To obtain lipophilic membranes, either $1 \%(\mathrm{w} / \mathrm{w})$ stearic acid (Col/St acid) or $0.25 \%$ $(\mathrm{w} / \mathrm{w})$ cetyl alcohol $(\mathrm{Col} / \mathrm{CetAl})$ or a mixture of $0.125 \%$ (w/w) cetyl alcohol and $0.125 \%$ (w/w) cholesterol $(\mathrm{Col} / \mathrm{Cet} \mathrm{Al} / \mathrm{Chol})$ were dissolved in collodium, respectively. Aliquots of $200 \mu \mathrm{L}$ solutions were placed one-sided on dry rings of the filter paper and were left to dry for $24 \mathrm{~h}$. The amount of the abovementioned substances applied to separate membranes after drying was $0.055 \mathrm{~g}$, on average.

Formulations. In donor chambers, $2 \mathrm{~g}$ of either hydrogel or emulsion $(\mathrm{o} / \mathrm{w})$ containing $0.5 \%(\mathrm{w} / \mathrm{w})$ of niacinamide was placed. Each experiment was performed in triplicate.

\section{Electrical impedance}

The electrical impedance of all the lipid membranes including human skin was measured using an LCR meter 4080, which was operated in parallel mode with an alternating frequency of $120 \mathrm{~Hz}$ (error at $\mathrm{k} \Omega$ values $<0.5 \%$ ). For the measurement, the donor compartment of the Franz diffusion cell was filled with the acceptor phase, and then the tips of the stainless-steel probes were carefully immersed in the PBS solution ( $\mathrm{pH} 7.4$ ) in the donor and acceptor compartments of the diffusion cell. Only membranes that produced an electrical resistance above 8 $\mathrm{k} \Omega$ were used for penetration study. The resistances observed in our study was comparable to the resistance of human skin (25).

The thickness of the lipid membranes which were prepared was measured with the optical microscope Junalumar with DELTA OPTICAL Camera Viewer.

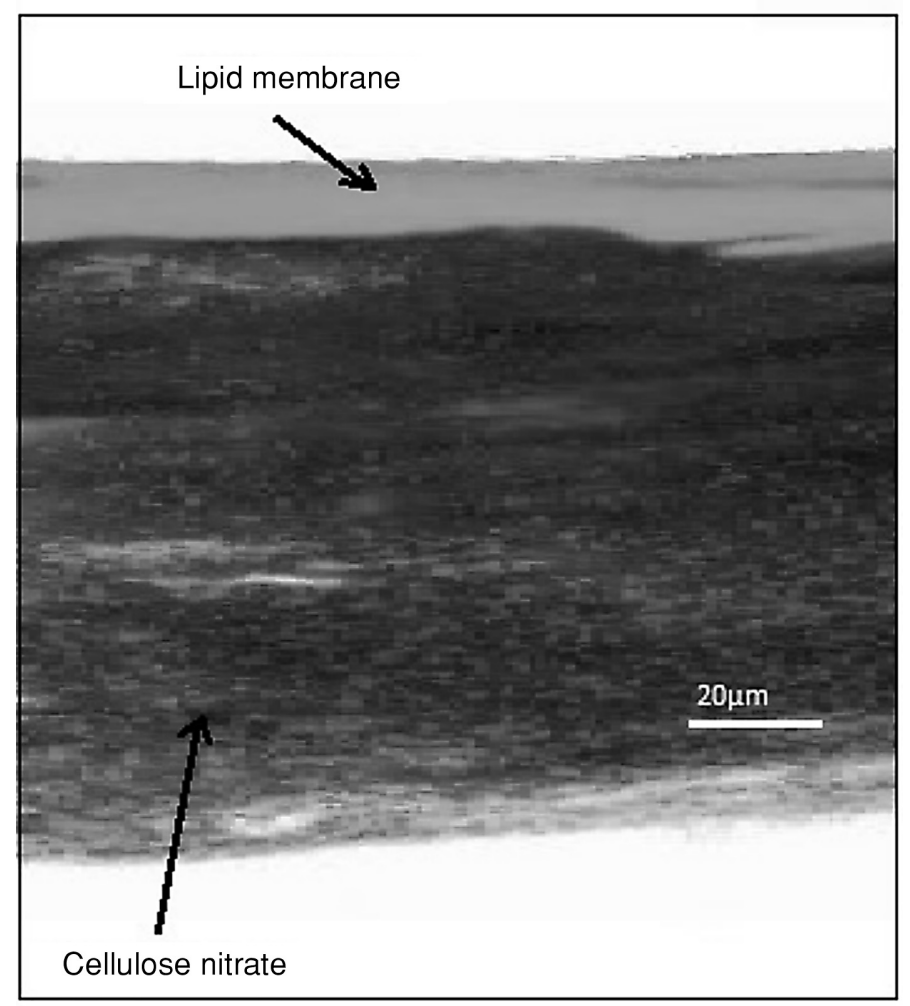

Figure 2. Micrograph of a cross-section of the artificial lipid membranes $[\mathrm{Col} / \mathrm{Ceth} . \mathrm{Al} / \mathrm{Chol}]$ on the cellulose nitrate filter. 


\section{HPLC analysis}

The determination of niacinamide concentrations in analyzed samples was performed by high performance liquid chromatographic (HPLC) method with spectrophotometric detection (Knauer, Berlin, Germany). The compounds were separated isocratically at room temperature on $125 \times 4 \mathrm{~mm}$ ID column filled with Hyperisil ODS, particle size $5 \mu \mathrm{m}$. The mobile phase consisted of $0.005 \mathrm{M}$ aqueous potassium dihydrogen phosphate solution and acetonitrile ( $95: 5$ by vol.). Aliquots of $20 \mu \mathrm{L}$ of analyzed samples were injected onto the column. The determination was performed at a wavelength of $280 \mathrm{~nm}$.

To determine niacinamide lipophilicity, the octanol-water partition coefficient $\left(\log \mathrm{K}_{\mathrm{o} / \mathrm{w}}\right)$ at $\mathrm{pH}$ 5.4 and 7.4 (identical to the $\mathrm{pH}$ of the acceptor fluid in the permeability experiment) was evaluated by a shake-flask method. Octanol was mixed with an equal volume of an adequate phosphate buffer containing various niacinamide concentrations. The mixture was then shaken on a rotating shaker SK0330-PRO (Chemland, Poland) for four hours. The octanol/water partition coefficient, being a measure of the lipophilicity of the examined compound, was calculated as a logarithm of the ratio of niacinamide concentration in octanol to its concentration in a phosphate buffer of a specific $\mathrm{pH}$. The results were presented as an average of analyzed samples con- centrations. For each applied niacinamide concentration three samples were collected for HPLC analysis.

\section{Statistical analyses}

Results were presented as arithmetical mean \pm standard deviation (SD). The results were evaluated using a one-way analysis of variance (ANOVA). For detailed data analysis, Tukey's post-hoc test was used. Probabilities $<0.05$ were considered to be statistically significant. To evaluate the significance of differences between samples collected after skin vs. artificial membranes penetration paired t-tests were applied. All determinations were performed in triplicate $(n=3)$.

\section{RESULTS}

Figure 2 presents the optical micrograph of the lipid membrane on the cellulose nitrate filter. The lipid layer was homogeneous without leak. The average thickness (measured from the three points separately) of the prepared lipid membranes was 9.7 $\pm 1.9 \mu \mathrm{m}(\mathrm{n}=3)$ (Fig. 2).

The octanol/water partition coefficients (log $K_{\mathrm{o} / \mathrm{w}}$ ) determined for two buffers of different $\mathrm{pH}$ were $-0.43 \pm 0.03$ and $-0.29 \pm 0.03$ for $\mathrm{pH} 5.4$ and 7.4 , respectively.
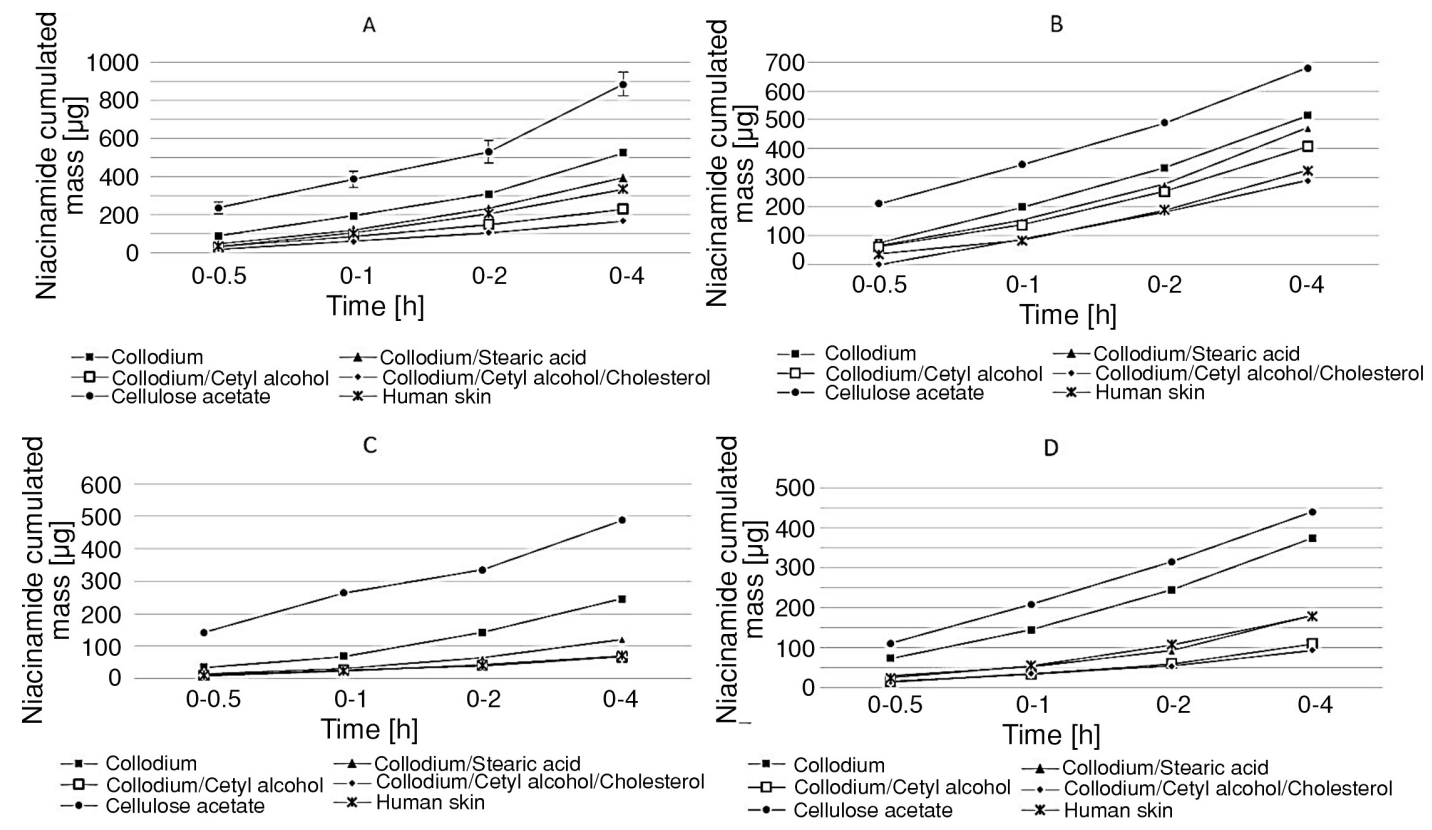

Figure 3 A-D. Comparison of mean cumulated mass of niacinamide penetrated from hydrogel (A, B) and emulsion (C, D) through artificial hydrophilic and lipophilic membranes as well as human skin into acceptor fluid pH 5.4 (A, C) and pH 7.4 (B, D) A. hydrogel - acceptor fluid pH 5.4 B. hydrogel - acceptor fluid pH 7.4 C. emulsion - acceptor fluid pH 5.4 D. emulsion - acceptor fluid pH 7.4. 
A

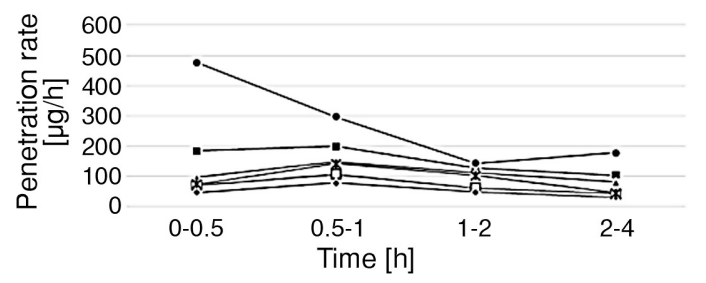

--COllodium
$-\square-$ Collodium/Cetyl alcohol $-\mathbf{-}-$ Collodium/Stearic acid $\begin{array}{lll}- & - \text { Collodium/Cetyl alcohol }- \text { - Collodium/Cetyl alcohol/Cholesterol } \\ -\bullet-\text { Cellulose acetate } & -\boldsymbol{*} \text { - Human skin }\end{array}$

C

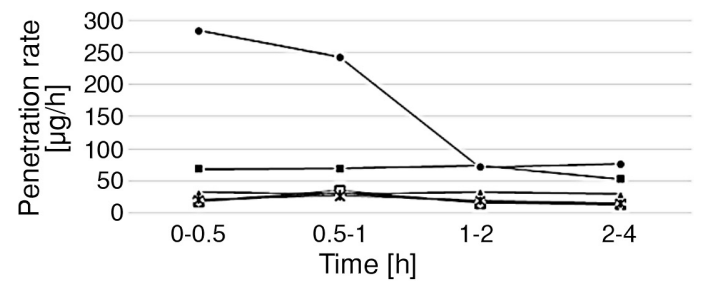

--Collodium $\quad \rightarrow$ Collodium/Stearic acid

- - Collodium/Cetyl alcohol -- Collodium/Cetyl alcohol/Cholesterol --Cellulose acetate

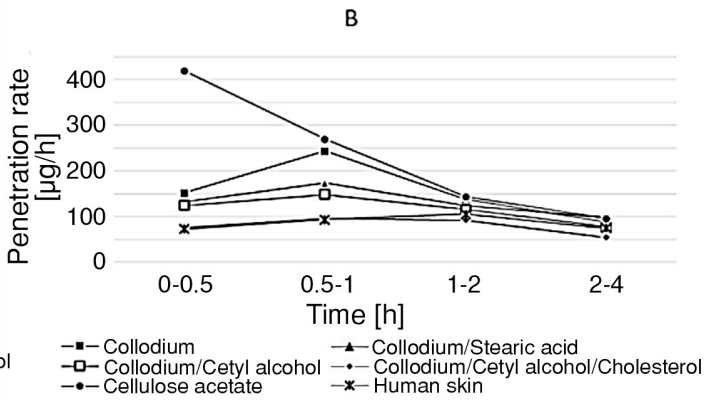

D

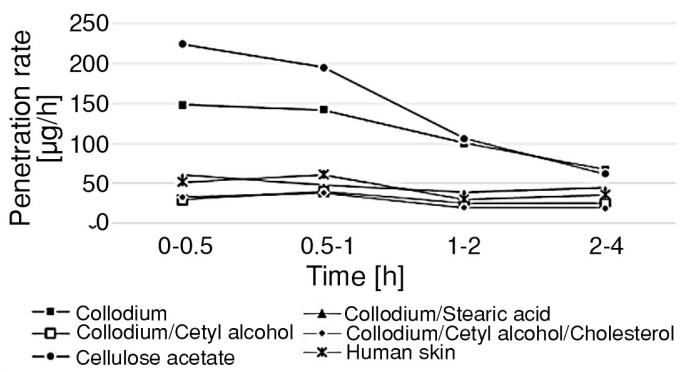

Figure 4 A-D. Comparison of the mean penetration rate of niacinamide from hydrogel (A, B) and emulsion (C, D) through artificial hydrophilic and lipophilic membranes as well as human skin into acceptor fluid pH 5.4 (A, C) and pH 7.4 (B, D) A. hydrogel - acceptor fluid $\mathrm{pH}$ 5.4 B. hydrogel - acceptor fluid $\mathrm{pH}$ 7.4 C. emulsion - acceptor fluid pH 5.4 D. emulsion - acceptor fluid $\mathrm{pH} 7.4$.

Figure 3 A-B shows the comparison of NA cumulated mass (in $\mu \mathrm{g}$ ) permeating at specific intervals up to $4 \mathrm{~h}$. The results are presented separately for hydrogel and emulsion taking into account different $\mathrm{pH}$ of the acceptor fluid (5.4 and 7.4). The extent of niacinamide penetration through studied membranes, expressed as cumulated mass, can be ranked as follows: the significantly highest transport was observed via CA membrane, followed by Col, whereas the lower permeation was found via $\mathrm{Col} / \mathrm{St}$ acid, $\mathrm{Col} / \mathrm{CetAl}, \mathrm{Col} / \mathrm{CetAl} / \mathrm{Chol}$ membrane, and human skin. After application of hydrogel, the highest total NA amount was observed after penetration through $\mathrm{CA}(883 \pm 62 \mu \mathrm{g}$ for $\mathrm{pH} 5.4$ and $679 \pm 21 \mu \mathrm{g}$ for $\mathrm{pH}$ 7.4 of the acceptor fluid). High penetration was also found at both pH 5.4 and $\mathrm{pH} 7.4$ (524 \pm 31 and $515 \pm$ $22 \mu \mathrm{g}$, respectively) if Col membrane was applied.

The cumulative niacinamide penetration of 394 $\pm 4 \mu \mathrm{g}$ NA observed at pH 5.4 through lipophilic $\mathrm{Col} / \mathrm{St}$ acid membranes was comparable to penetration through human skin $(356 \pm 17 \mu \mathrm{g})$. The lowest permeation was observed through $\mathrm{Col} / \mathrm{CetAl}$ and $\mathrm{Col} / \mathrm{CetAl} / \mathrm{Chol}$ membranes $(232 \pm 7$ and $170 \pm 12$ $\mu \mathrm{g}$, respectively). At $\mathrm{pH} 7.4$ the total mass after $4 \mathrm{~h}$ collection was for $\mathrm{Col} / \mathrm{St}$ acid $472 \pm 8.2 \mu \mathrm{g}$, for $\mathrm{Col} / \mathrm{CetAl} 408 \pm 5 \mu \mathrm{g}$, for HS 325 $24 \mu \mathrm{g}$ and for Col/CetAl/Chol $291 \pm 17 \mu \mathrm{g}$ (Fig. 3 A, B).
In contrast, after emulsion application, a lower permeation of the studied ingredient was observed (Fig. 3 C, D). In this case, the compound permeated to a higher degree solely through hydrophilic membranes made of cellulose acetate. After $4 \mathrm{~h}$ of collection, the total amount in the acceptor fluid of $\mathrm{pH}$ 5.4 and 7.4 was $487 \pm 46 \mu \mathrm{g}$ and $440 \pm 30 \mu \mathrm{g}$, respectively, and differed significantly as compared to the remaining samples. Also, high penetration through Col membrane was found (246 $\pm 10 \mu \mathrm{g}$ and $374 \pm 7 \mu \mathrm{g}$ for $\mathrm{pH} 5.4$ and 7.4, respectively). After application of lipophilic membranes lower permeation was observed, the total niacinamide mass permeating via Col/St acid membrane was $121 \pm 3 \mu \mathrm{g}$ for $\mathrm{pH} 5.4$ and $182 \pm 12 \mu \mathrm{g}$ for $\mathrm{pH} 7.4$, through human skin $71 \pm 8 \mu \mathrm{g}$ at $\mathrm{pH} 5.4$ and $180 \pm 13$ at $\mathrm{pH}$ 7.4, across $\mathrm{Col} / \mathrm{CetAl}$ membranes $67 \pm 5 \mu \mathrm{g}$ at $\mathrm{pH}$ 5.4 and $111 \pm 12 \mu \mathrm{g}$ at $\mathrm{pH} 7.4$ and via $\mathrm{Col} / \mathrm{CetAl} / \mathrm{Chol} \mathrm{membranes} 67 \pm 2 \mu \mathrm{g}$ at $\mathrm{pH} 5.4$ and $95 \pm 2$. $\mu$ g at $\mathrm{pH} 7.4$ ) (Fig. $3 \mathrm{C}, \mathrm{D}$ ).

The rate of niacinamide permeation (defined as a mass of the penetrating compound in time unit $\mu \mathrm{g} \cdot \mathrm{h}^{-1}$ ) from hydrogel or emulsion through all types of the tested membranes, to the acceptor fluid of $\mathrm{pH}$ 5.4 and 7.4 is presented in Figure 4 A-D. Taking into account the individual forms of preparation, it was confirmed that from both formulations (hydrogel 
and emulsion) the fastest penetration was found during the first two time intervals $(0-0.5 \mathrm{~h}$ and $0.5-1 \mathrm{~h})$. This was particularly evident in the case of hydrophilic CA membrane, after application both hydrogel and emulsion (Fig. 4 A-D).

In Figure 5 A-D the hierarchical dendrogram for all types of tested membranes is presented. Diagrams are presented for separate cosmetic products and each $\mathrm{pH}$ of acceptor fluid. A similarity between human skin and lipophilic membranes were found. After applications of either emulsion or gel on lipophilic membranes group of similar properties were observed. In contrast, the penetration of niacinamide through a cellulose acetate membrane was different (Fig. 5).

Table 1 shows the comparison of the penetration niacinamide through different lipophilic and hydrophilic artificial membranes to human skin. In the case of lipophilic membranes, in most cases, similar penetration in comparison with human skin was shown (Table 1).

\section{DISCUSSION AND CONCLUSIONS}

The penetration of active substances applied to the skin is largely limited by stratum corneum barrier which contains a number of lipophilic substances that can influence the permeation of exogenous com- pounds, both therapeutics and cosmetics $(3,4,26)$. To evaluate in vitro permeation of compounds of interest, human or pig skins are quite often used (27).

In our study, the average thickness of the prepared lipid membranes was $9.7 \mu \mathrm{m}(n=3)$ and was comparable to stratum corneum lipid membranes observed by others (28).

To evaluate in vitro permeation of exogenous compounds, the application on human or animal skin seems to be the most correct model. However, as human skin is not easily available, a number of experimental models have been developed for the evaluation of in vitro penetration of specific components. These include, inter alia, the application of artificial lipophilic and hydrophilic membranes. The former may correspond to the lipophilic character of stratum corneum, whereas the latter mimics the deeper layers of the epidermis and the skin. The properties of applied artificial membranes should resemble the function of the skin barrier. As a first assumption, to predict penetration of certain compounds via biological membranes partition coefficient octanol/water could be used (26).

In our study, the permeability of niacinamide through artificial membranes was dependent mainly on the character of the membrane. However, lipophilicity of active compounds should also be taken into account. As previously mentioned, the
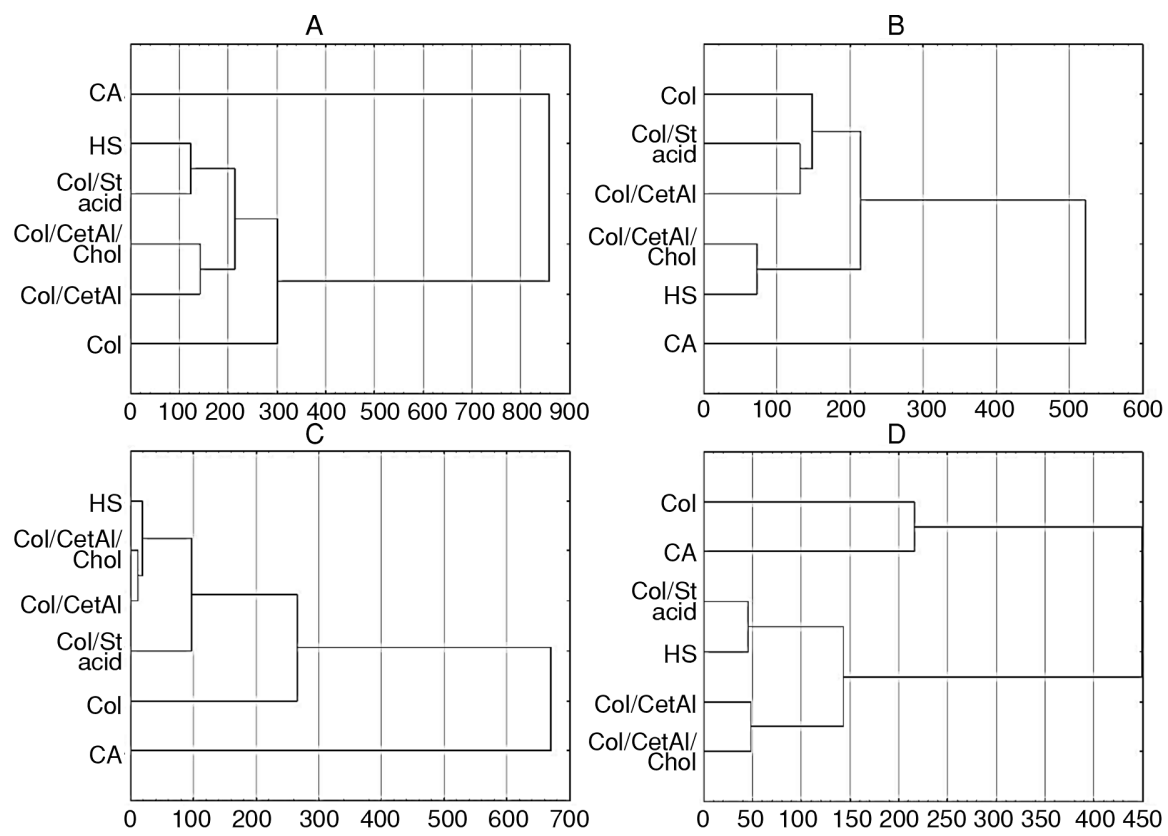

Figure 5 A-D. Hierarchical dendrogram of mean cumulated mass of niacinamide permeated from hydrogel (A, B) and emulsion (C, D) through artificial hydrophilic and lipophilic membranes and human skin into acceptor fluid of pH 5.4 (A, C) and pH 7.4 (B, D). A. hydrogel - acceptor fluid pH 5.4 B. hydrogel - acceptor fluid pH 7.4 C. emulsion - acceptor fluid pH 5.4 D. emulsion - acceptor fluid pH 7.4. 
Table 1. Comparison of cumulated in vitro penetration of niacinamide from gel and emulsion via different lipophilic and hydrophilic artificial membranes versus human skin into acceptor fluid of $\mathrm{pH}$ of 5.4 and 7.4.

\begin{tabular}{|c|c|c|c|c|c|}
\hline \multirow[t]{2}{*}{ Vehicle } & \multirow[t]{2}{*}{$\begin{array}{l}\mathrm{pH} \text { of } \\
\text { acceptor } \\
\text { fluid }\end{array}$} & \multirow[t]{2}{*}{ Membrane } & \multicolumn{3}{|c|}{$\begin{array}{c}\text { Significance of differences between } \\
\text { penetration through the artificial } \\
\text { membrane and human skin }\end{array}$} \\
\hline & & & $\mathrm{t}$ & $\mathrm{p}$ & \\
\hline \multirow[t]{10}{*}{ Gel } & 5.4 & Cellulose acetate & 4.5476 & 0.0199 & \\
\hline & & Collodium & 3.7772 & 0.0325 & \\
\hline & & Collodium + stearic acid & 2.5107 & 0.0869 & NS \\
\hline & & Colodium + cetyl alcohol & -1.9871 & 0.1411 & NS \\
\hline & & Collodium + cetyl alcohol + cholesterol & -2.4235 & 0.0939 & NS \\
\hline & 7.4 & Cellulose acetate & 7.1413 & 0.0057 & \\
\hline & & Collodium & 3.8376 & 0.0312 & \\
\hline & & Collodium + stearic acid & 3.3786 & 0.0431 & \\
\hline & & Colodium + cetyl alcohol & 4.6662 & 0.0186 & \\
\hline & & Collodium + cetyl alcohol + cholesterol & -1.1428 & 0.3360 & NS \\
\hline \multirow[t]{10}{*}{ Emulsion } & 5.4 & Cellulose acetate & 4.5827 & 0.0195 & \\
\hline & & Collodium & 2.5572 & 0.0834 & \\
\hline & & Collodium + stearic acid & 2.0804 & 0.1290 & NS \\
\hline & & Colodium + cetyl alcohol & -0.7056 & 0.5313 & NS \\
\hline & & Collodium + cetyl alcohol + cholesterol & -0.8650 & 0.4507 & NS \\
\hline & 7.4 & Cellulose acetate & 4.7307 & 0.0179 & \\
\hline & & Collodium & 3.7146 & 0.0339 & \\
\hline & & Collodium + stearic acid & -0.5450 & 0.6237 & NS \\
\hline & & Colodium + cetyl alcohol & -2.8480 & 0.0652 & NS \\
\hline & & Collodium + cetyl alcohol + cholesterol & -2.4709 & 0.0900 & NS \\
\hline
\end{tabular}

NS - no significance.

lipophilic nature of the compound can be defined as a logarithm of the partition coefficient between octanol and water or water solution, for example, an aqueous buffer of specific $\mathrm{pH}\left(\log \mathrm{K}_{\mathrm{o} / \mathrm{w}}\right)$. Niacinamide is a water-soluble compound $(18,23)$. This statement was also confirmed in our study, as log $K_{\mathrm{o} / \mathrm{w}}$ of the niacinamide was -0.46 for $\mathrm{pH} 5.4$ and -0.29 for $\mathrm{pH}$ 7.4. A similar value was obtained by Nicoli et al., in their experiment, the octanol-water partition coefficient was -0.40 (29). The solubility of niacinamide depends on the solvent. The highest solubility was found in the mixture of equal volumes of methanol and water (50:50), then in pure methanol, while the lowest in a phosphate buffer $\mathrm{pH}$ $7.3 \pm 0.1$ (18). As mentioned above, the lipophilicity of the compound is a substantiated factor influencing the degree of penetration of an active substance through hydrophobic stratum corneum. So, more lipophilic substances should usually better permeate through hydrophobic membranes $(15,30)$.
Uchida et al. evaluated the passage of active substances of various lipophilicity $\left(\log K_{\mathrm{o} / \mathrm{w}}\right.$ from -0.9 to 3.5) through three different types of membranes (Strat- $\mathrm{M}^{\mathrm{TM}}$, human skin, mouse skin), and found a correlation between $\mathrm{K}_{\mathrm{o} / \mathrm{w}}$ and the permeability coefficient $(\log \mathrm{P})$ of the applied substance penetrating through membranes: an increase of $\log \mathrm{P}$ value in the case of all applied membranes correlated with lipophilicity enhancement (26).

In our study, hydrophilic niacinamide permeated better through hydrophilic membranes: its total mass in an acceptor fluid measured after four hours of collection was significantly higher as compared to the mass penetrated through lipophilic membranes both at pH 5.4 and 7.4 of the acceptor fluid), regardless of the vehicle. The highest penetration was also observed through CA membranes, in this case, the cumulated mass was even several times higher in comparison with lipophilic membranes including HS (Fig. 3 A-D). 
The largest amount of niacinamide permeated from the hydrogel $(883 \mu \mathrm{g})$. This result may suggest better penetration through the deeper layers of the epidermis of hydrophilic nature (Fig. 3 A-D). Haque et al. stated that vitamin $B_{3}$ penetrated better through monolayer silicone membranes, however, the smaller dose was applied $\left(5 \mu \mathrm{L} / \mathrm{cm}^{2}\right)$. Its larger quantities, higher than $20 \mu \mathrm{L} / \mathrm{cm}^{2}$, permeated better through multi-layer membranes such as natural pigskin, it might be related to the type of applied membranes, either synthetic or natural ones. In our study, niacinamide permeated better through monolayer CA membrane. As compared to human skin, niacinamide penetration was two times higher on average. Similar results were also obtained by Haq et al. They found markedly higher penetration of diclofenac, hydrocortisone and caffeine via cellulose acetate membranes as compared to human skin. Cellulose acetate formed simple porous membranes - its pore size is greater than that of human skin, so this can lead to a greater permeability as compared to natural membranes (27).

The permeability rate, defined as a quantity of studied compound penetrated in time interval and expressed in $\mu \mathrm{g} \cdot \mathrm{h}^{-1}$, from hydrogel and emulsion through all types of synthetic membranes as well as through human skin, at both $\mathrm{pH}$ of the acceptor fluid ( $\mathrm{pH} 5.4$ and $\mathrm{pH} 7.4$ ) was the highest in the first two time intervals, i.e. $0-0.5 \mathrm{~h}$ and $0.5-1 \mathrm{~h}$. This rate was particularly evident in the case of penetration through hydrophilic, i.e. cellulose acetate membrane (Fig. 4 A-D).

Stahl et al. evaluated the permeability of ibuprofen ( $5 \%$ by weight) from three formulations, i.e. gel, emulsion and solution of $\mathrm{pH} 7.4$ through the skin of cow's udders. During $4 \mathrm{~h}$ study, they found the fastest permeability of the tested drug applied in the form of hydrogel as compared with emulsion (W/O) and water solution (31). A similar tendency was observed in our study, the highest niacinamide amount penetrated from hydrogel through a cellulose acetate membrane in the first hour of experiment, as compared with the later collection periods.

The vehicle contained active substance seems to be a significant factor that may affect properties of stratum corneum and thus the penetration of active substances to the skin. Depending on the type of formulation, an interaction may occur between the vehicle and the membrane (30). Moreover, the solubility of an active substance in a given vehicle seems to be another crucial factor (9). In our study, two types of formulations were used. Niacinamide permeated in a higher degree from hydrogel, while its permeability from the emulsion was lower (Fig. 3 A-D). Dias et al. evaluated the impact of lipophilic and hydrophilic excipients on the caffeine, benzoic acid and salicylic acid penetration through silicone membranes. The permeation degree of lipophilic substances (benzoic acid and salicylic acid) was higher from lipophilic vehicles as compared with penetration of hydrophilic compounds, i.e. caffeine (30). The ingredients of the lipid vehicle, such as paraffin, oils and fats, form an occlusion which can enhance the permeation. Typically hydrophilic carriers such as hydrogels may increase the quantity of water and skin hydration (32). In our study hydrogel contained glycerol was used. This compound can also act as a humectant, and then enhance the permeability of the tested active substances. Moreover, a high degree of skin hydration could lead to disrupt lipid ultrastructure of stratum corneum and to increase permeability as a result (27).

In contrast, the lower penetration of hydrophilic niacinamide from emulsion might be partly due to the presence of the lipid phase in that form of preparation. A number of artificial membranes, frequently applied in scientific experiments, are similar to natural taking into account permeation of compounds through them. This applies to such lipophilic membranes as Strat-M, as well as other membranes, contained ceramides, cholesterol, free fatty acid and silicone oil $(9,14)$. In our study penetration degree across hydrophobic membranes was comparable to human skin permeation. In cluster analysis, such membranes formed most frequently one cluster of similar properties (Fig. 5 A-D).

In conclusion, the permeation of active substances applied to skin in various types of formulation is mostly dependent on the type and properties of the vehicles.

In this study, to evaluate in vitro penetration, both artificial lipophilic membranes composed of, among others, stearic acid, cetyl alcohol, and cholesterol, as well as hydrophilic membranes, made of collodion (consisted mainly of cellulose nitrate) or cellulose acetate as well as human skin were used.

Niacinamide penetration through the artificial lipophilic membranes has been similar to the penetration through human skin in vitro, however, it has been lower as compared to monolayer hydrophilic membrane CA, Similar results were also obtained by Haque et al. (18) and Haq et al (27). Moreover, Jung et al. also confirmed higher penetration of hydrophilic substances including niacinamide through artificial CA membrane as compared to lipophilic made of polytetrafluoroethylene (33).

The proposed in our study method could be useful to the preliminary evaluation of penetration through biological membranes of compounds of dif- 
ferent polarity, however, the optimal penetration could be found for the unionized compounds.

These types of rather uncomplicated, easy to make lipo- and hydrophilic membranes may become useful tools, an alternative to natural skin, to predict the in vitro permeability of active components of topical preparations applied to the skin.

\section{Funding}

This work was supported by the Pomeranian Medical University in Szczecin [WNoZ-32601/S/14]

\section{Conflicts of interest}

The authors declare no conflict of interest.

\section{REFERENCES}

1. Klimowicz A., Farfał S., Bielecka-Grzela S.: J. Clin. Pharm. Ther. 32, 143 (2007).

2. Prausnitz M.R., Langer R.: Nat. Biotechnol. 26, 1261 (2008).

3. Baroni A., Buommino E., De Gregorio V., Ruocco E., Ruocco V., et al.: Clin. Dermatol. 30, 257 (2012).

4. Bolzinger M.A., Briançon S., Pelletier J., Chevalier Y.: Curr. Opin. Coll. Int. Sci. 17, 156 (2012).

5. Lam P.L., Gambri R.: J. Control. Rel. 178, 25 (2014).

6. Meuwissen M.E., Janssen J., Cullander C., Junginger H.E., Bouwstra J.A.: Int. J. Pharm. 238, 105 (1998).

7. Roberts M.S., Mohammed Y., Pastore M.N, Namjoshi S., Yousef S., et al.: J. Control. Rel. 247, 86 (2017).

8. Palac Z., Engesland A., Flaten G.E., ŠkalkoBasnet N., Filipović-Grčić J., et al.: J. Liposome. Res. 24, 313 (2014).

9. Karadzovska D., Riviere J.E.: Eur. J. Pharm. Sci. 50, 569 (2013).

10. Yu H., Wang Q., Sun Y., Shen M., Li H., et al.: PLoS One 10. http://doi.org/ 10.1371/journal. pone.0116502 (2015).

11. Zhang Y., Lane M.E., Hadgraft J., Heinrich M., Chen T., et al.: Int. J. Pharm. 556, 142 (2019).

12. Sinkó B., Garrigues T.M., Balogh G.T., Nagy Z.K., Tsinma O., et al.: Eur. J. Pharm. Sci. 45, 698 (2012).
13. Luo L., Patel A., Sinko B., Bell M., Wibawa J., et al.: Int. J. Pharm. 505, 14 (2016).

14. Simon A., Amaro M.I., Healy A.M., Cabral L.M., de Sousa V.P.: Int. J. Pharm. 512, 234 (2016).

15. Loftsson T., Konrádsdóttir F., Másson M.: Int. J. Pharm. 326, 60 (2006).

16. Ng S.F., Rouse J., Sanderson D., Eccleston G.: Pharmaceutics 2, 209 (2010).

17. Borges V.R., Simon A., Sena A.R., Cabral L.M., Sousa V.P.: Int. J. Nanomed. 8, 535 (2013).

18. Haque T., Lane M.E., Sil B.C., Crowther J.M., Moore D.J.: Int. J. Pharm. 30, 158 (2017).

19. Damian D.L.: Australas. J. Dermatol. 58, 174 (2017).

20. Walocko F.M., Eber A.E., Keri J.E., Al-Harbi M.A., Nouri K.: Dermatol. Ther. 30, doi: 10.1111/dth.12481 (2017).

21. Rolfe H.M.: J. Cosmet. Dermatol. 13, 324 (2014).

22. Emanuele E., Bertona M., Altabas K., Altabas V., Alessandri G.: Clin. Cosm. Invest. Dermatol. 5, 33 (2012).

23. Wohlrab J., Kreft D.: Skin Pharmacol. Physiol. 27, 311 (2014).

24. Badran M.M, Kuntsche J., Fahr A.: Eur. J. Pharm. Sci. 36, 511. (2009)

25. Kopečná M., Macháček M., Prchalová E., Štěpánek P., Drašar P., et al.: Pharm. Res. 34, 2097 (2017)

26. Uchida T., Kadhum W.R., Kanai S., Todo H., Oshizaka T., et al.: Eur. J. Pharm. Sci. 67, 113 (2015).

27. Haq A., Dorrani M., Goodyear B., Joshi V., Michniak-Kohn B.: Int. J. Pharm. 539, 58 (2018).

28. Čuříková B.A., Procházková K., Filková B., Diblíková P., Svoboda J., et al.: Int. J. Pharm. 534, 287 (2017).

29. Nicoli S., Zani F., Bilzi S., Bettini R., Santi P.: Eur. J. Pharm. Biopharm. 69, 613 (2008).

30. Dias M., Hadgraft J., Lane M.E.: Int. J. Pharm. 336, 108 (2007).

31. Stahl J., Wohlert M., Kietzmann M.: BMC Pharmacol. 11, 1 (2011).

32. Björklund S., Engblom J., Thuresson K., Sparr E.: Eur. J. Pharm. Sci. 50, 683 (2016).

33. Jung Y.J., Yoon J.-H., Kang N.G., Park S.G., Joeng S.H.: J. Pharm. Invest. 42, 271 (2012).

(C) 2020 by Polish Pharmaceutical Society. This is an open-access article under the CC BY NC license (c) (1) (8) (http://creativecommons.org/licenses/by-nc/4.0/). 